\title{
Blazar kinematics reloaded: Jet ridge line properties and evolution in the CJF
}

\author{
Marios Karouzos* \\ $+$ \\ Max-Planck-Institute fuer Radioastronomie, Germany \\ E-mail: mkarouzos@mpifr-bonn.mpg.de
}

\section{Silke Britzen}

Max-Planck-Institute fuer Radioastronomie, Germany

\section{Arno Witzel}

Max-Planck-Institute fuer Radioastronomie, Germany

\section{Anton Zensus}

Max-Planck-Institute fuer Radioastronomie, Germany

I. Physikalisches Institut, Universitaet zu Koeln, Germany

\section{Andreas Eckart}

I. Physikalisches Institut, Universitaet zu Koeln, Germany

Max-Planck-Institute fuer Radioastronomie, Germany

\begin{abstract}
Recent radio VLBI studies of the morphology and kinematics of individual BL Lac objects (e.g., 1803+784) have revealed a different kinematic scenario for these objects. Most, if not all, jet components appear to be stationary with respect to the core, while exhibiting strong changes in their position angles. We use the Caltech-Jodrell Bank flat-spectrum sample to test this new scheme, in particular probing a possible difference between BL Lac and quasar properties.
\end{abstract}

10th European VLBI Network Symposium and EVN Users Meeting: VLBI and the new generation of radio arrays

September 20-24, 2010

Manchester, $U K$

\footnotetext{
* Speaker.

${ }^{\dagger}$ Member of the International Max Planck Research School (IMPRS) for Astronomy and Astrophysics at the Universities of Bonn and Cologne.
} 


\section{Introduction}

Although observed in the minority of active galaxies $(\sim 5-15 \%$; Kellermann et al. 1989; Padovani 1993), extragalactic jets are some of the most pronounced morphological features in AGN research. VLBI observations enable the direct imaging of AGN jets and thus the study of their properties.

Jet kinematics, as studied through the investigation of distinct components, is currently explained in terms of the shock-in-jet model (Marscher \& Gear 1985), where the observed jet knots are manifestations of shocks propagating down the jet at relativistic speeds. Beaming and projection effects regulate the observed properties of the jets. There has been ongoing effort to distinguish whether the jet properties of different AGN types (e.g., QSOs, BL Lacs, Fanarrof-Riley, etc.) are just a result of orientation effects, or if additionally these objects have intrinsically different properties. Analysis of statistically significant samples of active galaxies have been of fundamental importance to this end (e.g., Ghisellini 1993; Vermeulen 1995; Taylor et al. 1996; Lister \& Homan 2005; Britzen et al. 2007b).

\subsection{Motivation}

S5 1803+784 is one of the several BL Lac objects (e.g., 0716+714, PKS 0735+178) recently found to exhibit a different kinematic scheme than other blazars (e.g., Britzen et al. 2009; Britzen et al. 2010a; Britzen et al. 2010b). All components of its inner jet (out to 12mas) appear to remain stationary with respect to the core. In addition, they show strong changes in their position angles, implying a prevailing movement perpendicular to the jet axis. The general classification of blazar is often used to describe members of both the BL Lac and QSO classes. This phenomenological unification comes into question in light of the investigation of the sources mentioned above.

We use the CJF sample to statistically investigate and assess the similarity, or divergence, of the kinematic and morphological properties between the two distinct sub-samples of QSOs and BL Lac objects in the CJF. We want to test whether BL Lac objects jet components indeed show slower apparent speeds with respect to their cores compared to their QSO counterparts. Furthermore we are interested in the phenomenon exhibited in S5 1803+784 of a, at times, very wide jet, as well as a strong evolution of that width.

\section{Data}

The CJF sample (Taylor et al. 1996) consists of 293 radio-loud active galaxies selected from three different samples (for details, see Britzen et al. 2007b). The sources span a large redshift range (see Fig. 1 in Britzen et al. 2008), out to a redshift of $z=3.889$ (1745+624). All the objects have been studied in the radio (e.g., Britzen et al. 2007b; Britzen et al. 2008) and in the X-rays (Britzen et al. 2007a), while the evolution of active galaxies, in the context of the merger-driven evolution scheme, has also been investigated using the CJF (Karouzos et al. 2010).

The work presented here is based on the kinematic analysis of the CJF sample (Britzen et al. 2007b; Britzen et al. 2008). An extensive observing campaign of all 293 CJF sources was undertaken using both the VLBA and the global VLBI array at 5GHz (see Britzen et al. 2007b for details). In total, 288 sources are considered and analyzed in the following sections. Of these, 196 
are classified as quasars, 49 as radio galaxies, 33 as BL Lac objects, and 10 are not classified. We will focus here on BL Lacs and QSOs.

\section{Tools}

In short we used the following measures: (1) apparent Jet Width, dP, (2) apparent Jet Width Evolution, $\Delta P$, and (3) apparent Jet Linear Evolution, $\Delta \ell^{1}$.

\subsection{Jet width, dP}

For an epoch $i$ and a jet consisting of $\mathrm{N}$ components characterized by their core separation and position angle $\left(r_{i}, \theta_{i}\right)$, we identify the components with the maximum and the minimum position angles. The apparent width of the jet $\mathrm{dP}$, measured in degrees, is then calculated as:

$$
d P_{i}=\theta_{i}^{\max }-\theta_{i}^{\min }
$$

while the error is calculated by the propagation of errors formula. By using the definition above, we seek to quantify the opening of the jet flow and identify the effect of a channel-like jet, as seen in the case of S5 $1803+784$.

\subsection{Jet width evolution, $\Delta P$}

The jet width evolution $\Delta P$, measured in degrees per unit time, is measured between two successive epochs $i$ and $(i-1)$. It is calculated as follows:

$$
\Delta P=\frac{d P_{i}-d P_{i-1}}{T_{i}-T_{i-1}}
$$

where $T_{i}$ denotes the time at epoch $i$, measured in years. We can also define the maximum jet width evolution as:

$$
\Delta P^{\max }=\max \left\{\Delta P_{1 \ldots i}\right\}
$$

This value is characteristic of each source and reflects the maximum potential width change of the jet flow for that source. The per unit time reflects a normalization for time that ensures a comparison between sources that have been observed during different time spans.

\subsection{Jet linear evolution, $\Delta \ell$}

We calculate the linear evolution across all available epochs and for all components, ultimately acquiring a value that reflects the total linear displacement of the whole jet ridge line. We use planeof-the-sky coordinates $\left(X_{i}, Y_{i}\right)$ to calculate the linear displacement of component $m$ between epochs $i$ and $(i+1)$ :

$$
l_{i}^{m}=\sqrt{\Delta X^{2}+\Delta Y^{2}} .
$$

To calculate the total displacement of the whole jet ridge line we then need to sum up over all components and all available observing epochs and account for both the different time span of

\footnotetext{
${ }^{1}$ All the above measures refer strictly to values projected onto the plane of the sky. For the sake of brevity, we shall drop the characterization of apparent for each of these values, although this will be implied throughout.
} 
observations, as well as the different number of components. Therefore we define the jet linear evolution as:

$$
\Delta \ell=\frac{\sum_{i-1} \sum_{m} l_{(i)}^{m}}{N\left(T_{i}-T_{1}\right)}=\frac{\ell}{N d T}
$$

where $\mathrm{N}$ here is the total number of components used across all the epochs.

$\Delta \ell$ can be seen as a mean jet component speed over all components and all available epochs.

By comparing sources at different redshifts one implicitly studies a different part of their jet. We therefore explicitly define a maximum core separation limit, above which components are not included in our investigation.

\section{Analysis and results}

As the jet length (expressed by the maximum distance of the outermost jet component identified in each source, across all available epochs) is used as a basis for the further analysis, we briefly discuss this first. BL Lacs show the shortest jets, both on average and in median values, compared to quasars ( $35 \pm 7 \mathrm{pc}$ and $17.3 \pm 2.7 \mathrm{pc}$, compared to $43 \pm 7 \mathrm{pc}$ and $27.1 \pm 2.4 \mathrm{pc}$ ). We can now define a maximum core separation limit. We use BL Lacs as the basis of our decision, adopting a limit of 40 parsecs. All components at separations larger than 40 parsecs with respect to the core are not included in the following analysis. There is a further reasoning for this limit: for $1803+784$, peculiar kinematics of the components is observed for the inner-most part of its jet (up to 12 mas), with most robust effects out to 6 mas. For this source's redshift, this translates roughly to ( $82 \mathrm{pc}$ ) $41 \mathrm{pc}$.

\subsection{Jet width, $P$, statistics}

Using a total of more than 500 epochs, we can calculate an average jet ridge line width for our sources. BL Lacs show significantly wider jet ridge lines (average value of $22.6 \pm 1.9^{\circ}$ ), compared to both QSOs $(11.9 \pm 0.4 \circ)$ and RGs $\left(16.7 \pm 1.0^{\circ}\right)$. A Student's t-test gives a significance of $>99.99 \%$ that BL Lacs and QSOs show different mean values. For a redshift bin of $[0,1]$ BL Lacs still show wider jets than both QSOs and RGs.

In Fig. 1 (left panel) we show the distribution of jet ridge line widths for BL Lacs, QSOs, and RGs, in the redshift bin $[0,1]$. It can be seen that all three classes show similar distributions, with their maxima situated at around 10 degrees. The distribution of BL Lac appears to be wider and extending to larger widths compared to QSOs. Quasars show a distribution much more confined to lower width values than BL Lacs.

We apply the two-sample Kolmogorov-Smirnoff (K-S) test to our data to see whether BL Lacs and QSOs show different jet ridge line width distributions. The K-S test gives a $0.7 \cdot 10^{-5}$ probability that these two sub-samples originate from the same parent distribution. We also compare the sub-samples of BL Lacs and QSOs in the redshift bin [0,1]. In this case the K-S test gives a probability of $1 \%$ that BL Lacs and QSOs are drawn from the same parent sample. We can conclude that indeed BL Lacs show a significantly different distribution of jet ridge line widths compared to QSOs. In both average and median values, BL Lacs show substantially wider jets than QSOs. 

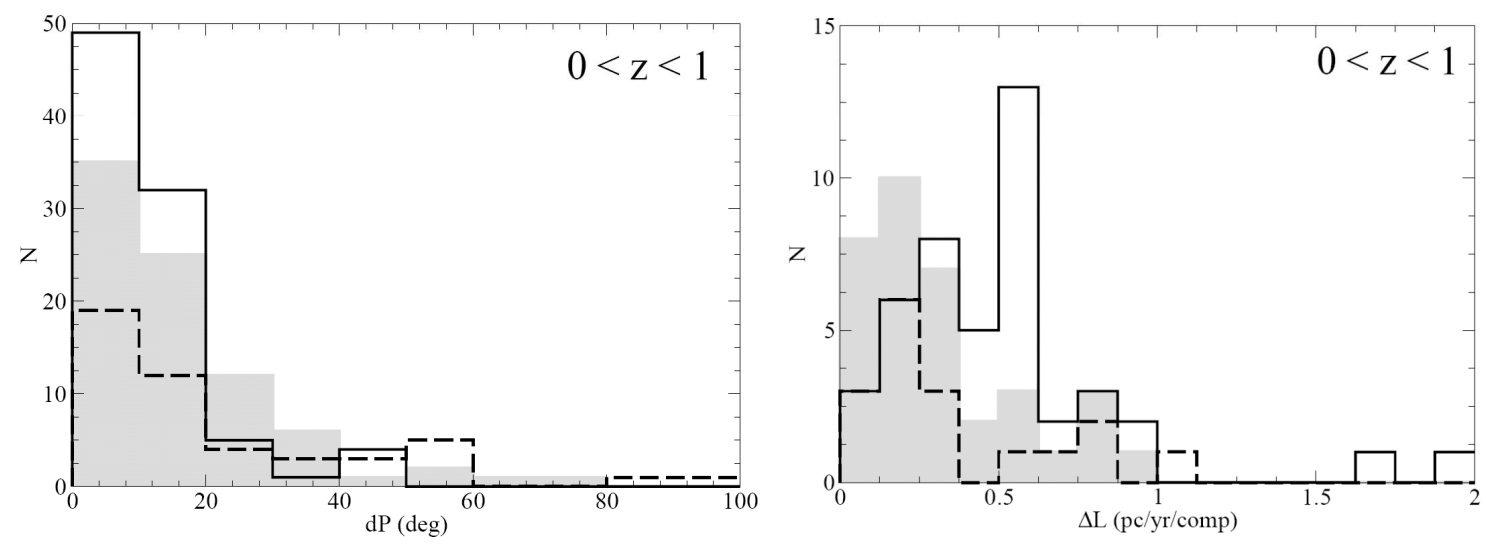

Figure 1: Absolute distributions of the jet ridge line width (left) and the total linear evolution (right) for QSOs (solid line), BL Lacs (dashed line), and RGs (grey blocks). For this histogram sources with redshifts in the $[0,1]$ bin are used.

\subsection{Jet width evolution, $\Delta P$, statistics}

To account for the effect of a changing number of jet components between epochs, we flag and exclude sources for which $N_{\text {comp }}$ between epochs varies by more than 1 . We compare the maximum values of the jet ridge line width evolution per source between BL Lacs and quasars for a redshift bin of $[0,1]$. BL Lac objects show the higher average and median values $\left(4.7 \pm 0.8^{\circ} / y r\right.$ and $3.6 \pm 0.5^{\circ} / y r$, compared to $3.5 \pm 0.4^{\circ} / y r$ and $2.42 \pm 0.20^{\circ} / y r$ for QSOs).

A K-S test is inconclusive concerning whether the jet width evolution distributions of BL Lacs and QSOs are significantly different. We can tentatively say that indeed the BL Lacs show on average larger changes in their jet ridge line width, as compared to QSOs.

\subsection{Jet linear evolution, $\Delta \ell$, statistics}

On average, BL Lacs show weaker jet ridge line evolution than QSOs $(0.37 \pm 0.05 \mathrm{pc} / \mathrm{yr} / \mathrm{comp}$ and $0.470 \pm 0.016 \mathrm{pc} / \mathrm{yr} / \mathrm{comp}$, respectively). Looking at the median values, BL Lacs show the least evolution compared to QSOs ( $0.236 \pm 0.026$ and $0.414 \pm 0.012$, respectively). For the $[0,1]$ redshift bin the behavior remains the same as before, with differences between BL Lacs and QSOs becoming more pronounced (however the errors also increase, given the smaller number of sources).

Taking into account only sources in the redshift bin [0,1] (see Fig. 1, right), quasars show a strong maximum around $0.63 \mathrm{pc} / \mathrm{yr} / \mathrm{comp}$, with a secondary maximum around $0.37 \mathrm{pc} / \mathrm{yr} / \mathrm{comp}$ (secondary to primary ratio $\sim 0.62$ ). In contrast, BL Lacs show their distribution maximum around $0.25 \mathrm{pc} / \mathrm{yr} / \mathrm{comp}$, with a secondary maximum around $0.87 \mathrm{pc} / \mathrm{yr} / \mathrm{comp}$ (ratio $\sim 0.33$ ). Quasars show a wider distribution of total linear evolution values, also showing the highest maximum values among all three types. For the whole sample (independent of redshift constraints), the K-S test gives a probability of $4.3 \%$ that BL Lacs and QSOs are drawn from the same parent population. For the $0<z<1$ sub-sample, we get a $5.4 \%$ probability from the K-S test that BL Lacs and QSOs stem from the same parent sample. These results give a positive answer as to whether BL Lacs show less absolute linear evolution of their jets compared to their QSO counterparts. 


\section{Discussion}

One obvious factor that should play a deciding role for the kinematic of jet components is the viewing angle under which a source is observed. Could this viewing angle difference explain the kinematic differences seen between BL Lacs and quasars? Naively speaking, assuming that jet components follow ballistic, linear paths, one can expect that, viewed at smaller viewing angles, the components are observed to cover smaller distances than if seen edge-on. Therefore the slower speeds, and hence smaller total linear evolution of their jet ridge lines, observed for BL Lacs can be explained in terms of a viewing angle effect.

An additional mechanism or effect must be introduced to produce a wide jet. There are a number of models that could readily explain this, usually employing either a precession of the jet axis, or by assuming that the components follow non-ballistic and non-linear paths (e.g., Steffen et al. 1995; Gong 2008). In these models, jet components follow highly curved trajectories, which, viewed face-on, would give the impression of a wider distribution of jet component position angles.

\section{Conclusions}

We provide detailed insight concerning the morphology and evolution of AGN jet ridge lines. The statistical investigation of the CJF sources lends strong support to the different kinematic scenario recently seen in a number of BL Lac objects. BL Lac objects do not follow the kinematic paradigm, widely accepted for blazars, of outward superluminally moving jet components. BL Lacs appear to evolve their jet ridge lines (with respect to the core) less than the other source classes, hence indicating a slower flow in their jets. On the other hand, they show significantly wider jets, that change their widths more strongly, than both QSOs and RGs.

Acknowledgments: MK was supported for this research through a stipend from the International Max Planck Research School (IMPRS) for Astronomy and Astrophysics. This research has made use of the NASA/IPAC Extragalactic Database (NED) which is operated by the Jet Propulsion Laboratory, California Institute of Technology, under contract with the National Aeronautics and Space Administration.

\section{References}

Britzen, S., Brinkmann, W., Campbell, R. M., et al. 2007a, A\&A, 476, 759

Britzen, S., Kam, V. A., Witzel, A., et al. 2009, A\&A, 508, 1205

Britzen, S., Kudryavtseva, N. A., Witzel, A., et al. 2010a, A\&A, 511, A57+

Britzen, S., Vermeulen, R. C., Campbell, R. M., et al. 2008, A\&A, 484, 119

Britzen, S., Vermeulen, R. C., Taylor, G. B., et al. 2007b, A\&A, 472, 763

Britzen, S., Witzel, A., Gong, B. P., et al. 2010b, A\&A, 515, A105+

Ghisellini, G. 1993, Advances in Space Research, 13, 587

Gong, B. 2008, MNRAS, 389, 315

Karouzos, M., Britzen, S., Eckart, A., Witzel, A., \& Zensus, A. 2010, A\&A, 519, A62+

Kellermann, K. I., Sramek, R., Schmidt, M., Shaffer, D. B., \& Green, R. 1989, AJ, 98, 1195

Lister, M. L. \& Homan, D. C. 2005, AJ, 130, 1389

Marscher, A. P. \& Gear, W. K. 1985, ApJ, 298, 114

Padovani, P. 1993, MNRAS, 263, 461

Steffen, W., Zensus, J. A., Krichbaum, T. P., Witzel, A., \& Qian, S. J. 1995, A\&A, 302, 335

Taylor, G. B., Vermeulen, R. C., Readhead, A. C. S., et al. 1996, ApJS, 107, 37

Vermeulen, R. C. 1995, Proceedings of the National Academy of Science, 92, 11385 